\title{
EL CORAZÓN FRÁGIL. EL DOLOROSO DESPERTAR MORAL DEL DR. EDGAR PASCOE
}

\section{The fragile heart. The painful moral awakening of Dr. Edgar Pascoe}

María Eugenia D'OTTAVIO CALLEGARII'; María Silvia D'OTTAVO CALLEGARI²; Alberto Enrique D'OTTAVIO CATTANI $^{3}$

${ }^{1}$ Universidad del Gran Rosario, Rosario (Santa Fe) (Argentina); ${ }^{2}$ Facultad de Derecho de la Universidad Nacional de Rosario, Rosario (Santa Fe), (Argentina); ${ }^{3}$ Facultad de Ciencias Médicas y Consejo de Investigaciones, Universidad Nacional de Rosario, Rosario (Santa Fe) (Argentina).

Correo electrónico: aedottavio@hotmail.com

Fecha de recepción: 21 de enero de 2020

Fecha de aceptación: 11 de febrero de 2020

Fecha de publicación: 15 de diciembre de 2020

\section{Resumen}

El corazón frágil es una miniserie británica, estrenada en 1996. Dado que este drama médico ofrece, entre otras, una variedad de posibilidades de debate sobre facetas deontológicas y actitudinales para médicos en particular y profesionales del área de la salud en general resulta más que recomendable apreciar las varias lecturas que permite.

El objetivo de este trabajo es abordarla y presentar algunas de tales posibilidades.

Palabras clave: corazón; frágil; medicina; deontología; actitudes.

\section{Abstract}

The fragile heart is a British miniseries, released in 1996. Since this medical drama offers, among others, a variety of possibilities for debate on deontological and attitudinal features for physicians in particular and professionals in health area in general is more than recommended appreciating the various readings it allows.

The aim of this paper is to address it and present some of those possibilities.

Key words: heart; fragile; medicine; deontology; attitudes. 


\section{Introito}

El corazón frágil es una miniserie de 3 o 4 episodios (según el país), de origen británico, estrenada en 1996.

Dado que este drama médico ofrece, entre otras, una variedad de posibilidades de debate sobre facetas deontológicas y actitudinales para médicos en particular y profesionales del área de la salud en general resulta más que recomendable apreciar las varias lecturas que permite.

El objetivo de este trabajo es abordarla y presentar algunas de tales posibilidades.

\section{Sinopsis argumental}

Edgar Pascoe, prestigioso cardiocirujano y, a la vez, cardiópata, frío y desdeñoso, enmarca su proceder en la medicina basada en pruebas biológicas y en una ética altamente permisiva consigo mismo.

Está casado con Lileth, compasiva y amplia de miras, quien ejerce como médica general de cabecera y atiende, entre otros, a un anciano que fuera su médico y el de su familia muchos años atrás. Tras su fallecimiento, le es entregado todo lo que él coleccionara en lugares adonde viajaba para mantener la mente abierta y alejada de dogmas.

Edgar y Lileth, inmersos en una crisis matrimonial, tienen mellizos: Nicola, médica ambiciosa, inescrupulosa y favorita del padre pues sigue sus pasos, y Daniel, minusvalorado por éste y por su hermana ya que abandonó los estudios médicos y trabaja como empleado en Devlin Medical, empresa de telemedicina.

Más allá de los vaivenes sentimentales entre los miembros de este singular grupo familiar, el tema principal se centra en un viaje a China de padre e hijo, viaje que, tras la pantalla de conferencias a cargo del primero y de la presentación de nuevos recursos tecnológicos por el segundo, esconde el principal objetivo: pingües negocios en un mercado numeroso.

Poco antes de la partida, Pascoe induce a uno de sus pacientes, con concretos reparos al respecto, a someterse a un bypass cardíaco. Fallecido durante la intervención, su viuda, quien se dejó convencer por el cardiólogo clínico derivador (Dr. Mlchael Denton) y el cardiocirujano, los denuncia ante el colegio profesional acusándolos de doloso reparto de tarifa.

Confiado en que sus contactos londinenses les evitarán problemas, se traslada al Lejano Oriente. Allí, a poco de llegar, enfrenta tres situaciones conflictivas: las objeciones a su posicionamiento médico por parte de un profesional chino de visión más holística (Dr. Chen), una cirugía a practicar de manera clandestina a uno de los líderes chinos Wan Pu (convenida previamente en Londres) y el encuentro con una médica local (Dra. Zhao Quing) que le pide interceda ante dicho líder para que cese el tráfico ilegal de órganos extraídos de disidentes políticos y delincuentes ejecutados por el régimen.

Tres hechos alteran su irreductibilidad afectiva y profesional en destacables secuencias cinematográficas:

1. Solicitada por Pascoe como una de sus colaboradoras en la cirugía, su hija se reúne con él en China y le revela no sólo que se ha apropiado de datos científicos obtenidos por un compañero para realizar una ventajosa publicación personal para su futuro en The Lancet sino que, además, le propone una artimaña para desembarazarse de aquél.

2. Impactado por ello, padece un severo acceso coronario que lo lleva a reencontrarse con el Dr. Chen en una habitación del hotel. Éste acude en su carácter de médico del alojamiento y soslayando los comentarios sardónicos del cardiocirujano acerca de su manera alternativa de pensar y actuar, le narra que quedó reducido a tal condición profesional por manifestarse en contra del tráfico ilegal de órganos de prisioneros. Asimismo, le relata la historia de un médico australiano y de su misteriosa muerte a fin de ejemplificarle la influencia de la psiquis en el acto médico. Esta historia, estructurada por Chen, 
aparece fragmentada desde el primer episodio y la sombra de su protagonista interpela a menudo de modo premonitorio a Pascoe en sus duermevelas.

3. La entrevista previa a la operación con Wan Pu donde trata infructuosamente de plantear las aberraciones éticas del régimen y su impotente intervención ulterior que hace que lo fuercen a regresar de inmediato a Londres Allí, tras su movilizadora experiencia oriental, se enfrentará con la denuncia que se hiciera contra él y Denton, con el futuro de sus hijos y el de su matrimonio y con su propio porvenir.

Finalmente, cabe acotar que en el episodio final Pascoe hace referencia indirecta a la ya por entonces conocida psico-neuro-inmuno-endocrinología.

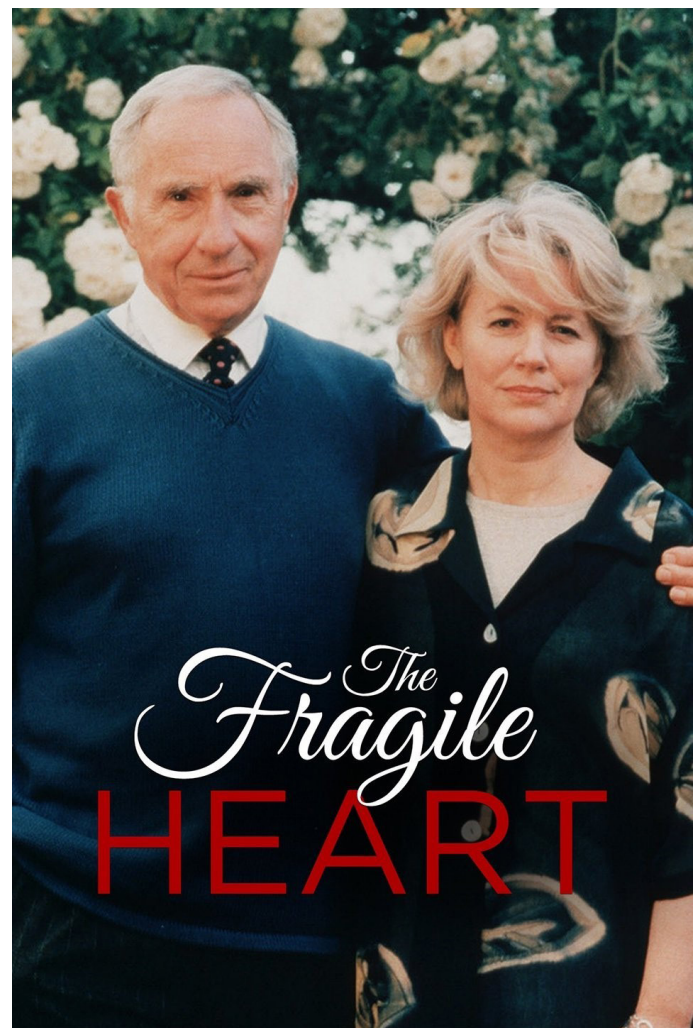

Cartel original.

\section{Ficha Técnica}

Título: The fragile heart (miniserie en 3 episodios). País: Reino Unido.

Año: 1996.

Director: Patrick Lau.

Música: Gavin Greenaway.

Fotografía: Nicholas D. Knowland.

Montaje: Neil Thompson.

Guión: Paula Milne.

Intérpretes: Nigel Hawthorne (Edgar Pascoe); Dearbhla Molloy (Lileth Pascoe); Helen McCrory (Nicola Pascoe); Dominic Mafham (Daniel Pasco); Robert Langdon Lloyd (Michael Denton); Sylvia Chang (Zhao Quing); Hon-Lam Pau (Wan Pu); Sai-Kit Yung (Chen).

Color: Color.

Duración: 48:56 min (Episodios 1 y 2); 47:24 min (episodio 3) y 50:28 min (episodio 4).

Género: drama médico.

Idioma original: inglés.

Sinopsis: «Edgar, un cirujano cardíaco, se enfrenta a un dilema ético sobre el abuso de los derechos humanos en China, donde encabeza una delegación médica mientras se enfrenta con su esposa Lileth, quien se siente cada vez más atraída por los métodos holísticos de curación que se practican allí» (IMDb).

Productora: Carnival Film \& Television.

Enlaces:

https://www.imdb.com/title/tt0115177/

\section{A modo de colofón}

Si bien cosechó varias nominaciones durante 1997 y obtuvo premios como mejor serie dramática (Festival de televisión de Banff 1997) y al mejor actor, guión y producción (BAFTA), recibió oportunamente tanto críticas ponderativas como ásperas ${ }^{1}$.

El corazón frágil es un filme de suma pertinencia en el grado y posgrado pues pone el acento en aspectos a evitar durante el ejercicio profesional: reparto de tarifa entre médicos, mercantilización de la Medicina, plagio de trabajos científicos,

Rev. Med. Cine. 2020; 16(4), 315-318 Ediciones Universidad de Salamanca / 
defectos actitudinales (arrogancia, sentimiento de omnipotencia y omnisciencia que conduce al control de pacientes), fanatismo científico y predominio de la tecnociencia, entre los salientes.

Alude, también, a la necesidad de empatía y de no olvidar los derechos humanos.

Habla, asimismo, sobre la relevancia de practicar, dentro de lo posible, una medicina integral y personalizada y de mantener mente abierta hacia otras culturas ancestrales sin caer obviamente en ingenuas creencias por el simple hecho de tal antigüedad.
Todos los episodios se cierran con un tren circulando hacia un punto de fuga enfocado desde abajo a modo de recordatorio de la sorprendente historia del médico australiano y, sobre todo, del doloroso despertar moral del Dr. Edgar Pascoe.

\section{Referencias}

1. Sutcliffe T. Review. Culture Section. Independent [Internet]. Thursday 7 November; 1996.

\begin{tabular}{|c|c|}
\hline & $\begin{array}{l}\text { María Eugenia D’Ottavio Callegari. Licenciada en Psicopedagogía (Univer- } \\
\text { sidad de San Martín - sede Rosario -). Ha trabajado en la clínica y en insti- } \\
\text { tuciones educativas. Docente de Metodología de la Investigación Científica, } \\
\text { Psicología Institucional y Psicología Genética en la Carrera de Licenciatura } \\
\text { en Psicopedagogía (Universidad del Gran Rosario, Rosario, Argentina). Inte- } \\
\text { grante de equipos de investigación psicopedagógica y coautora de trabajos } \\
\text { educacionales-culturales. }\end{array}$ \\
\hline & $\begin{array}{l}\text { María Silvia D’Ottavio Callegari Licenciada en Derecho. Facultad de Derecho, } \\
\text { Universidad Nacional de Rosario, Rosario, Argentina. Profesora Superior y } \\
\text { docente. Abogada relatora en la Corte Suprema de Justicia de la Provincia } \\
\text { de Santa Fe, Rosario, Argentina. Coautora de trabajos sobre su especialidad } \\
\text { y educacionales-culturales. }\end{array}$ \\
\hline & $\begin{array}{l}\text { Alberto Enrique D’Ottavio Cattani. Medico, Doctor, Ex Catedrático de } \\
\text { Histología y Embriología. Profesor Honorario de la Facultad de Ciencias } \\
\text { Medicas e Investigador Superior de la Carrera del Investigador Científico, } \\
\text { Universidad Nacional de Rosario, Rosario, Argentina. Autor y coautor de } \\
\text { libros, capítulos de libros y trabajos biológicos y educaciones-culturales. } \\
\text { Integrante de Bancos de Evaluadores nacionales e internacionales. Miembro } \\
\text { de comités evaluadores y editoriales de revistas nacionales y extranjeras. } \\
\text { Ex Consultor en Educación Médica de la Oficina Panamericana de la Salud } \\
\text { (Organización Mundial de la Salud). }\end{array}$ \\
\hline
\end{tabular}

Rev. Med. Cine. 2020; 16(4), 315-318 Ediciones Universidad de Salamanca / @@@

J. Med. Mov., 2020; 16(4), 315-318 